J. Astrophys. Astr. (1996) 17, 183-197

\title{
Making the Transition from Newton to Einstein: Chandrasekhar's Work on the Post-Newtonian Approximation and Radiation Reaction
}

\author{
Bernard F. Schutz \\ Max Planck Institute for Gravitational Physics, The Albert Einstein Institute, \\ Potsdam, Germany.
}

\section{Chandrasekhar the relativist}

Chandra first became interested in general relativity in the early 1930s. He had already discovered that white dwarfs had a maximum mass, and Eddington had pointed out that this would imply that stars of a larger mass could collapse to black holes. $^{1}$ Eddington thought this idea so abhorrent that he felt forced to dismiss Chandra's work on white dwarfs; this story is described elsewhere in this volume. But Chandra drew the opposite conclusion. He realised that he would need to understand general relativity in order to follow the implications of his discovery to their natural conclusions (Chandrasekhar [unpublished autobiographical memoirs]).

Nevertheless, he did not study relativity immediately. Discouraged by the evident hostility towards general relativity shown by many prominent physicists in the 1930s (in his private memoirs (Chandrasekhar [unpublished autobiographical memoirs]) he mentions Bohr in particular), and believing that general relativity had already proved to be a "graveyard of many theoretical astronomers", he steered a different course. In the 1950s, when he again thought of the subject, he remarked that astronomers doing relativity "were prone to play for high stakes", while his own "approach to science was more conservative"; this perception was later to be borne out by his work on the post-Newtonian approximation, and in particular in deriving the reaction effects of gravitational radiation, where his conservatism helped him avoid mistakes that had been made by others. He finally took up his interest in relativity in the $1960 \mathrm{~s}$, when he had such a strong scientific reputation that, as a friend said to him, "What can you lose?".

But once he started in relativity, he never left it. After the 1960s, Chandra worked almost exclusively on problems in general relativity. Even in the 1960s, his impatience to finish other research projects and get on with relativity is evident in his memoirs. He repeatedly refers to "distractions" that prevent him from working full-time on relativistic problems; among these distractions are, surprisingly, some

\footnotetext{
${ }^{1}$ The term 'black hole' was, of course, not used by Eddington; it was coined by John Wheeler in the 1960s.
} 


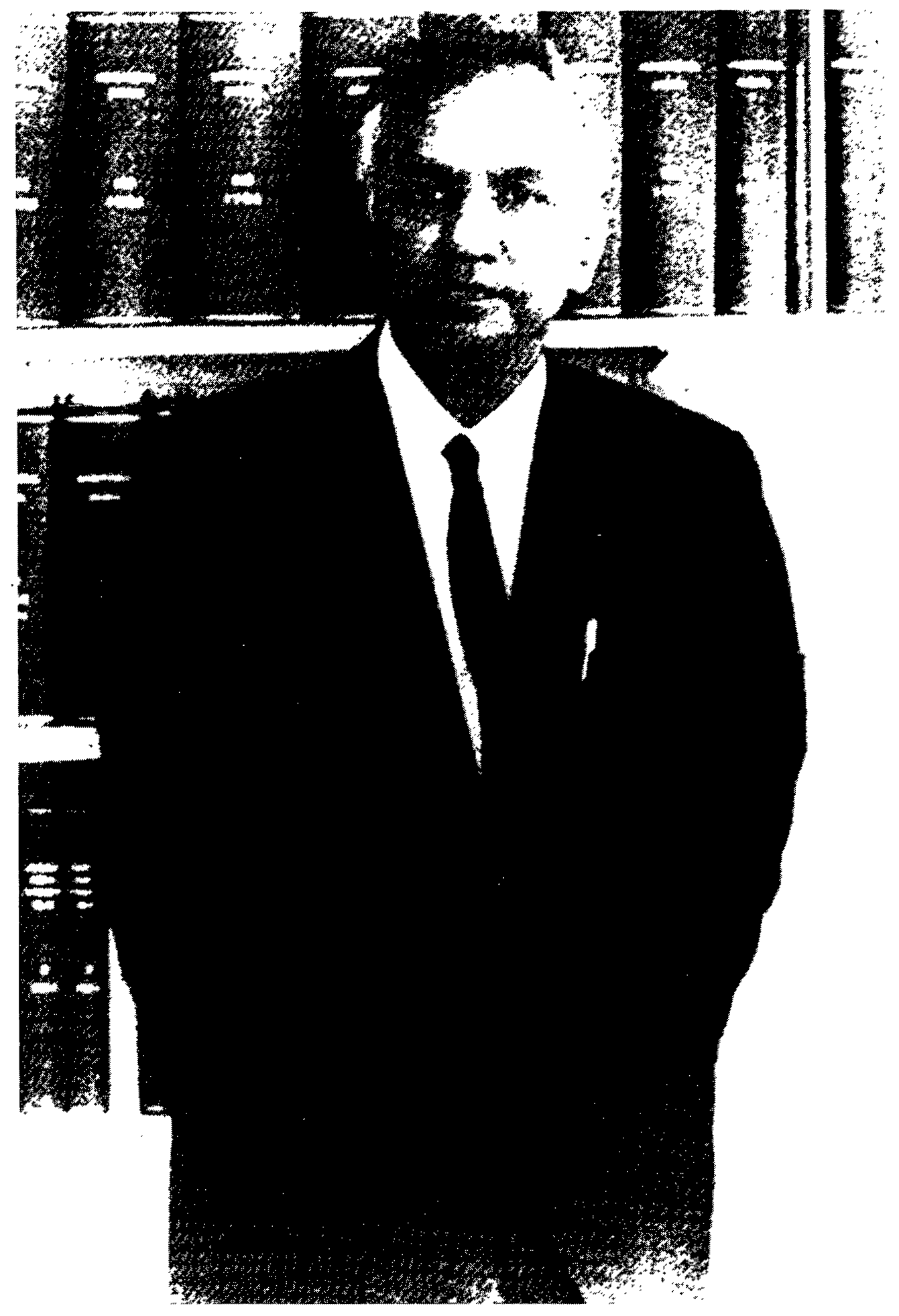

(C) Indian Academy of Sciences - Provided by the NASA Astrophysics Data System 
of his best-known work, such as the last stages of his work on the figures and stability of rotating homogeneous bodies in Newtonian gravity, and completing his book Ellipsoidal Figures of Equilibrium (Chandrasekhar 1969). His heart was in general relativity, which he had characteristically been learning by teaching courses on it while he finished up his other interests, and he was impatient to get to grips with new problems in it.

The first problem Chandra chose was to study the pulsation of a spherical star in general relativity. It proved surprisingly tractable, and it immediately had an impact on astronomy in showing that quasars were unlikely to be single supermassive stars. His work on this is described in the article by John Friedman in this volume; and there is an interesting comment on it in Thorne's foreword to one of the volumes of Chandra's collected papers (Thorne 1990).

Having gained confidence from this first project, Chandra wanted a more farreaching and fundamental goal to shoot for. He chose a problem that had defied solution for more than 40 years: how does the emission of gravitational radiation affect the emitting system, in particular when the system is self-gravitating? Many relativists had studied this problem, called radiation reaction. Some had provided partial answers, others had got completely wrong results. The situation was so confused that it had led some to doubt the reality of gravitational radiation, or at least the possibility of associating some kind of conserved energy with gravitational waves. This confusion was very unsatisfactory from both the physical and astrophysical points of view, and Chandra must have seen that there was a huge unexplored territory in astronomy waiting for him if he could clear up the confusion.

It was characteristic of Chandra's approach to physics that he saw this problem as one that he could solve by a careful step-by-step approach. He would start with the Newtonian limit of general relativity and introduce successively higher-order corrections until he came to the place where radiation reaction could be found. He would develop the post-Newtonian expansion to general relativity in complete detail.

No one before Chandra had attempted the post-Newtonian approximation for continuous bodies in such an exhaustive way. It was well known in relativity that the effects of radiation reaction could be expected to manifest themselves at a high order in such an expansion, but essentially every previous approach to finding them used one device or another to simplify the calculation. It was in handling these simplifications that most previous work had gone wrong. Chandra decided not to try tricks, but rather to trust in his ability to carry the calculation to a high order without getting lost in its complexity.

Chandra introduced no major new tools or conceptual breakthroughs in his calculation. Nor were his calculations particularly polished; some of the more awkward features of the work were to lead him later into controversy. Chandra studied previous work carefully, until he knew what methods to emulate, and what errors to correct. But essentially his method would have been available to anyone. No one before, however, had attempted to push it all the way to completion. In this he was true to his perception, quoted above, that he approached problems more 
conservatively than others. Previous work on this problem had aimed high, going for the radiation terms directly in one way or another, and failing. His approach was to keep his sights firmly on algorithmic calculations that he was confident would eventually lead him to the answer.

\section{Post-Newtonian relativity}

\subsection{Background}

I shall not review the history of approximate treatments of the equations of motion here. Excellent reviews can be found in Damour (Damour 1987) and the very recent study by Kennefick (1996 [preprint]). But it is useful to discuss briefly some of the simplifications that had gone wrong in earlier attempts at the radiation-reaction problem in relativity.

Radiation reaction is well understood in electromagnetism, where in a lowvelocity expansion of the equations of motion of a charged body, the reaction effects arise at order $(v / c)^{3}$. Put another way, the effective reaction force on a radiating charged body is proportional to the third time derivative of its position, or equivalently to the first derivative of its acceleration. ${ }^{2}$ All attempts at radiation reaction in general relativity started with lessons learned from the electromagnetic problem. This included using simplifications that worked successfully in electromagnetism.

The most common simplification was to treat the bodies as point-particles. This works well in electromagnetism, despite the fact that the electromagnetic self-field of a point charge is infinite on the body, and this self-field contains an infinite self-energy. By either absorbing the self-energy into the mass of the body, or using the radiative Green function introduced by Dirac (1938) (which is a trick that I will return to in the next paragraph), it is possible to show that radiation reaction acts on a point charge only at third order (Jackson 1962); there are no higherorder corrections. When the point-particle method was tried in general relativity, however, it ran into troubles with non-linearity. The infinite self-energy creates higher-order corrections to the Newtonian gravitational field that themselves get arbitrarily strong near the point mass, and handling these is delicate. In general relativity, it turns out to be possible to develop the post-Newtonian approximation by assuming the body is compact, and treating the gravitational field outside it as if the body were a point-mass.

In fact, Fock (1959), Peres (1960) and Peters (1964) had obtained the correct reaction formula in essentially the correct ways, but because the point-particle approach had also yielded incorrect answers in other hands, their papers were not universally accepted. ${ }^{3}$ Transparently successful ways of doing this were eventually

\footnotetext{
${ }^{2}$ Time derivatives in a slow-motion approximation raise the order of quantities by one power of $v$, so three time derivatives of a term like the position of a body produce a term of order $(v / c)^{3}$.

${ }^{3}$ In particular, Chandra was not aware of Peters' work before he completed his own, and he may not have been aware of Peres' work either.
} 
developed by, among others, Damour (1983), Walker and Will (1980), Walker (1984), and Anderson (1975), but these came after Chandra's work. ${ }^{4}$

The other simplification was to try to skip intervening orders in the expansion and calculate only the radiation-reaction terms. This is a trick that is closely related to Dirac's method for the point charge. In general relativity, as in electromagnetism, the fundamental equations are invariant under time-reversal, so that any dissipative effect, like the loss of energy to radiation, must be put in explicitly in some way. The conventional point of view ${ }^{5}$ is that the field that one must use is the particular solution of the field equations that has no radiation coming in to the system from the distant past; this is the solution that, in a linear theory like electromagnetism, follows from the retarded Green function. In nonlinear general relativity, one effectively puts in time-asymmetry by adopting a boundary (or asymptotic) condition of no incoming radiation on the field.

This condition is easy to implement in a linear field theory, such as in electromagnetism or in linearised gravitation. In linearised theory, radiation reaction turns up in a small-velocity expansion of the metric tensor (its 00 component) at order $(v / c)^{5} .^{6}$ Its implementation in a nonlinear field theory is not so straightforward, and in general relativity this has been a subject of considerable debate (Ehlers 1980). One way to isolate the radiation-reaction terms while ignoring all the others (thereby skipping lower orders) is to write the solution of the field equations using the retarded Green function and then expand it and the equations of motion in powers of $v / c$, keeping only the odd powers. Done correctly, this trick does work, but by the time Chandra began it had not been done correctly.

A related but more sophisticated version of this trick is to use the method of matched asymptotic expansions to find the terms in the equation of motion that couple directly to the outgoing radiation far away. This method was applied to the problem by the late William R. Burke and his Ph.D. supervisor, Kip Thorne (Burke 1969; Thorne 1969). Interestingly, they began their work after Chandra embarked on the full post-Newtonian calculation, and they reached their goal of

${ }^{4}$ Damour began his work completely unaware of Chandra's calculations; Walker \& Will and Anderson had studied the full history and tried to correct previous errors.

${ }^{5}$ There are other points of view, for example that the time-asymmetry arises statistically from random initial data (Schutz 1980). This subject is part of the physics of the arrow of time (Landsberg 1982).

${ }^{6}$ It is no mystery that this is at a higher order than in electromagnetism. The electromagnetic radiation reaction force at order $v^{3}$ is proportional to $d^{3}\left(\int \rho x^{i} d^{3} x\right) / d t^{3}$, where $\rho$ is the charge density. Because charge is conserved, this is equivalent to $d^{2}\left(\int \rho v^{i} d^{3} x\right) / d t^{2}$. The same result is true in linear gravity, provided we take $\rho$ to be the mass-density. But the integral $\int \rho v^{i} d^{3} x$ in this case is the total momentum of the system, which is conserved. (In general relativity, since all energies and momenta radiate, one must consider radiation from the entire mechanical system, not just one piece of it.) Its time-derivatives therefore vanish, and there is no gravitational radiation reaction at this order. At order $v^{5}$, the reaction forces in both electromagnetism and linear gravity depend on $d^{5}\left(\int \rho x^{i} x^{j} d^{3} x\right) / d t^{5}$, with $\rho$ suitably interpreted. This argument was well-understood in relativity by the time Chandra began his work. 
radiation reaction (skipping lower orders) a year before he did, largely because of the "distractions" referred to earlier, which frustrated Chandra's progress on the calculation. Nevertheless, Burke himself was not entirely convinced of the correctness of his result until Chandra derived an equivalent result. Many other relativists have viewed the Burke-Thorne method with some uneasiness (Ehlers $e t$ al. 1976), although in fluid dynamics the method of matched asymptotic expansions is an accepted tool that leads to derivations of important and experimentally testable results.

\subsection{The post-Newtonian expansion}

Against this background, Chandra felt that he would only have confidence in a full, order-by-order calculation. This was inevitably complicated because of the nonlinearity of Einstein's equations. When the system is self-gravitating and basically well-described by Newtonian gravity, orders in nonlinearity are related to orders in velocity. The virial theorem implies that the gravitational potential $\Phi$ should be of the same order as $v^{2}$, so that reaction terms from linear theory will not be the only $(v / c)^{5}$ terms. Terms of order $(v / c)^{3}\left(\Phi / c^{2}\right)$ and $(v / c)\left(\Phi / c^{2}\right)^{2}$ will be just as large, and must be considered. In addition, it could in principle happen that the nonlinearities would create reaction effects at lower order, say of order $(v / c) \Phi$. This would have to be looked for.

At the lowest order (zero-order in our way of counting), the expansion starts with the Newtonian equations. What can we say about the post-Newtonian expansion? The first observation is the one we made earlier, namely that Einstein's equations are, like the rest of classical physics, invariant under time reversal. So we expect the equations of motion to expand only in even powers of $v$, unless we introduce explicitly time-asymmetric terms in, say, the initial conditions or the boundary conditions. An initial non-zero velocity will introduce terms linear in $v$ into the metric components, but Chandra was only interested in the equations of motion, and these momentum-dependent metric terms affect the motion only by coupling to the velocity of the body, so that they appear as order $v^{2}$ corrections to the equations of motion. So the first non-trivial correction to the equation of motion would be of order $(v / c)^{2}$, or of order $\Phi$. This is called the first post-Newtonian order, denoted $1 \mathrm{pN}$. Post-Newtonian nomenclature numbers the orders according to their order beyond the Newtonian one in a nonlinearity expansion in $\Phi$.

It turns out that there are no nontrivial terms in the equation of motion at order $v^{3}$, so the next corrections are at the second post-Newtonian $(2 \mathrm{pN})$ order. However, there will be terms of order $v^{5}$, where we expect radiation reaction, so this was Chandra's goal: $2 \frac{1}{2} \mathrm{pN}$ order. 


\subsection{Chandra's work}

\subsubsection{First post-Newtonian order}

Chandra began working on the post-Newtonian approximation in spherical symmetry, where the equations are simplest. He found in this way the post-Newtonian dynamical instability of white dwarfs and radiation-dominated stars (Chandrasekhar 1964), but he soon turned to the full equations, without assumptions of symmetry. Chandra began with the $1 \mathrm{pN}$ equations of motion for a perfect fluid (Chandrasekhar 1965). He adopted his own choice of gauge, not equivalent to the de Donder gauge that had been used by most workers before him. He chose a gauge in which he could make the equations of motion look as similar to those of the Newtonian case as possible. He showed that the metric and the motion of the body could be adequately described at this order by a few potentials that satisfied generalisations of the Poisson equations. Chandra was particularly concerned to ensure that his post-Newtonian theory was physically reasonable and complete at this level of approximation, so he derived (by inspection) expressions for the energy and angular momentum densities, including gravitation, and showed that they were conserved as a result of the equations of motion at this order. The existence of these conservation laws also demonstrated, as he expected, that there was no effect of gravitational radiation at $1 \mathrm{pN}$ order.

\subsubsection{Second post-Newtonian order}

After a delay of about four years, during which he worked largely on uniformdensity ellipsoids, Chandra finally returned to the problem to develop, in the same systematic way, the much more complicated $2 \mathrm{pN}$ order of approximation. $\mathrm{He}$ worked on this with his Ph.D. student Yavuz Nutku (Chandrasekhar \& Nutku 1969). Again he chose a gauge that he felt was appropriate. Determining that the theory so far still obeyed conservation of energy and angular momentum was necessary but, in view of the complexity of the equations, it was not possible for him to determine the form of the conserved energy and angular momentum simply by inspection. Chandra realised that he could derive the conservation laws systematically from the Landau-Lifshitz pseudotensor, expanded to a suitable order. He justified this in a separate paper (Chandrasekhar 1969), where he showed that this method gave the same results at $1 \mathrm{pN}$ order as he had obtained by inspection. In the $2 \mathrm{pN}$ paper with Nutku, the same method led to the $2 \mathrm{pN}$-order conservation laws. Again, there was no radiation to this order.

The $2 \mathrm{pN}$ equations are of course very unwieldy, and Chandra never used them except as a way of showing that there was nothing left out on the road to radiationreaction order. Nevertheless, Chandra's insight into the key role of the LandauLifshitz tensor at this order was to prove crucial to his obtaining the right results for radiation-reaction. However, this step did give rise to considerable difficulties for other relativists who wanted to follow Chandra on this road. This is because 
Chandra's formal expressions for some of the metric terms at this order were divergent.

These divergences came about because Chandra chose a gauge in which, presumably, he felt he could make the equations look as similar as possible to the Newtonian equations. In this gauge, metric corrections played the role of higherorder gravitational potentials, satisfying certain Poisson-like differential equations. The derivatives (with respect to time and space variables) of these potentials contributed to the forces in the equations of motion. These potentials incorporated in part the nonlinearity of the theory, so the sources in their Poisson equations involved, for example, higher-order corrections to the mass-density, including the mass associated with the gravitational potential energy of the system. At the $1 \mathrm{pN}$ level of approximation, the gravitational potential energy can be localised in the volume containing the perfect fluid, for example in a term like $\rho \Phi / c^{2}$. But at the $2 \mathrm{pN}$ level, the sources for some of the potentials are distributed over all space. While most of them fall off rapidly near infinity, some do not, with the result that the solutions for some of these potentials are formally infinite. This infinity, which Chandra does not comment on in his papers, occurring at an order before the one at which we find radiation reaction, naturally cast doubt on the validity of Chandra's approximation. I shall describe below the way later work cleared up the doubts.

\subsubsection{Radiation reaction order}

Immediately after finishing the $2 \mathrm{pN}$ work, Chandra turned to the equations at radiation-reaction order, $2 \frac{1}{2} \mathrm{pN}$ order, working with another Ph.D. student, Paul Esposito (Chandrasekhar \& Esposito 1970). Here the final equations are simpler, since the messy conservative forces of the field appear at even orders. But the derivation of these equations is delicate, because one must apply an outgoingradiation condition on equations that are a near-zone approximation to the full solution, and this is where much of the previous work on the problem had gone wrong. Chandra read the previous literature extensively, and was attracted to the work of Trautman (1958), who had shown that, if one cast the field equations in the form of wave equations, wrote down solutions using retarded Green functions, and did a near-zone expansion of the result, then one would have a near-zone solution that incorporated the information that the solution was a retarded one and that the gravitational waves (which were not explicitly calculated in this approach) would be outgoing.

Trautman had, nevertheless, not obtained the correct answer, and after some thought Chandra knew why: Trautman had used, as his source for the solutions, only the stress-energy of the fluid system, and Chandra knew that he had to replace this with the full Landau-Lifshitz pseudotensor. This pseudotensor contained the gravitational stress-energy as well, and Chandra understood that this was essential to getting the correct result. The equivalence principle tells us that general relativity treats all energies in the same way, and this means that the gravitational radiation from a system will depend on the lowest-order Newtonian gravitational potential 
energy (resident in the Landau-Lifshitz pseudo-energy) as much as on, say, the kinetic energy of the fluid source. ${ }^{7}$

In order to incorporate the effects of retardation, Chandra had to alter his method substantially at this order; he chose the de Donder gauge in order to get wave equations, and he had to include in his near-zone expansion new terms from the expansion of the retardation parts of the solutions for the metric. He points out that he is free to adopt the de Donder condition at this order while keeping the previous gauge choices at lower order, so in his method, as contrasted with other approaches, he really uses wave equations for the first time only at this order. This is an unusual approach, not adopted by anyone else, but it is not inconsistent. In this way, Chandra and Esposito calculated the metric and equations of motion by close analogy with Trautman's attempt, but with the Landau-Lifshitz source.

This approach, while physically reasonable, raised another technical problem, but this one does not seem to have led to much criticism. Chandra found - for the same reason as at $2 \mathrm{pN}$ order, namely the extended nature of the source for his Poisson integrals - that there was one Poisson equation for a metric term, where the source was (in the near zone) a constant in space. The solution of such an equation again diverges at infinity. Unlike the divergent terms at $2 \mathrm{pN}$ order, Chandra worried about this point and felt he had to address it.

Chandra devised an intriguing method to get around this problem: he required that his metric solution be defined only in what was effectively a distributional sense. That is, he required that the metric give a finite integral when multiplied by any $C^{\infty}$ function that fell off near infinity faster than any inverse power of $r$. Using this, he found an expression for the metric that was well-defined, albeit divergent as $r \longrightarrow \infty$. However, and importantly, he did not need this metric outside the fluid bodies. It was used to calculate the radiation-reaction force on the system, where this metric term was always multiplied by the mass-density of the fluid itself, which was a function in the $C^{\infty}$ class defined by Chandra.

\subsubsection{The radiation reaction result}

This is where Chandra reached his goal, a goal that had eluded relativists for decades: the radiation-reaction force in a self-gravitating system. In the context of the time at which Chandra performed this calculation, it seems to me that his key insight was to combine Trautman's boundary condition with the Landau-Lifshitz pseudotensor. This was natural to him because he had laboured carefully over the lower orders, where the Landau-Lifshitz complex provided the conserved energy and angular momentum. Chandra's self-described "conservative" approach to the calculation had paid off: he had learned important lessons from the lower-order calculations.

\footnotetext{
${ }^{7}$ Others before Chandra, including Landau and Lifshitz themselves and also Fock, had understood that one must include the gravitational stresses to get the correct radiation at infinity, but they had not attempted the near-zone problem of radiation reaction.
} 
Of course, Chandra knew by the time he derived the reaction force that Burke and Thorne had already found an expression for it by the method of matched asymptotic expansions (Burke 1970; Thorne 1969). Chandra's expression was not identical to the force derived by Burke and Thorne, but it did give the same energy dissipation. This is another matter that Chandra does not comment on in his papers. However, there is a gauge transformation that makes it identical to the simpler Burke-Thorne result, which I quote here. The effects of radiation reaction at this order can be completely described by incorporating into the equations of motion a correction to the Newtonian potential of the form (Misner et al. 1973)

$$
\Phi_{\text {react }}=-\frac{1}{5} \frac{d^{5} \Psi_{j k}}{d t^{5}} x^{j} x^{k}
$$

where the symbol $I_{j k}$ stands for the reduced or trace-free quadrupole tensor of the Newtonian mass distribution,

$$
\Psi_{j k}=I_{j k}-\frac{1}{3} \delta_{j k} I_{n n}, \quad I_{j k}=\int \rho x_{j} x_{k} d^{3} x .
$$

By changing gauge one can find other forms for this that are equivalent, and some have proved useful in other problems.

\section{Influence, controversy and a re-assessment}

The completion of Chandra's post-Newtonian radiation-reaction work initially attracted attention because it gave, by a completely independent method, a result equivalent to that of Burke and Thorne. This gave astrophysicists confidence that general relativity was physically reasonable and well-behaved, that energy and angular momentum radiated in gravitational waves was correctly balanced by a loss from the energy and angular momentum of the radiating system. There were at least two immediate applications: Chandra's own discovery of the completely unexpected radiation-induced non-axisymmetric instability in rotating stars (described by John Friedman elsewhere in this volume), and the realisation that cataclysmic binary systems can be regulated by the competing effects of the radiation of angular momentum in gravitational waves (which brings the stars closer together) and the transfer of angular momentum from one star to another by mass flows (which in these systems drives the two stars apart) (Faulkner 1971).

There was a further direct influence of Chandra's post-Newtonian work, which was the development of the Parametrized Post-Newtonian (PPN) framework for describing a wide class of relativistic theories. The development of space physics and radio astronomy offered new opportunities for testing relativity, and it was initially not clear what the meaning of an experimental result would be if it contradicted a prediction of general relativity. It was clear that any reasonable theory of gravity must reduce to Newton's theory in the appropriate limit. Therefore the first 
deviations that would distinguish one theory from another would arise at the postNewtonian level. Theorists developed a framework for describing a large family of relativistic theories of gravity parameterizing their post-Newtonian predictions.

The first effort in this direction was by Kenneth Nordtvedt (Nordtvedt 1967), who in 1968 developed a PPN framework based on the Einstein-Infeld-Hoffman (EIH) point-particle approach. This was generalised to include fluids by Thorne and Will, who were directly motivated by what Thorne had learned about Chandra's post-Newtonian approach during a year-long visit to Chicago. Will (1981) used Chandra's perfect-fluid post-Newtonian equations as the basis for his PPN framework, developed as his PhD work for Thorne. ${ }^{8}$ The PPN formalism of Will, Thorne, and Nordvedt has become the framework for describing the results of tests of general relativity, initially for solar system experiments, but later also for the important Hulse-Taylor binary pulsar, which came shortly after the PPN framework was developed (Will 1981).

The Hulse-Taylor binary pulsar, announced in 1974 (Hulse \& Taylor 1975), was soon recognized as potentially the most important application of post-Newtonian theory: it was quickly realised that post-Newtonian effects on the orbits of the two stars would be observable through radiation-reaction order, and that the system would be a test of the correctness of general relativity's description of gravitational radiation. This proved to be so successful that the discoverers were awarded the Nobel Prize in Physics in 1993.

The prospect of testing post-Newtonian theory against the Hulse-Taylor system raised two kinds of questions about Chandra's post-Newtonian work. The first was whether it applied to the orbital motion of the two neutron stars in the HulseTaylor binary system even though it clearly did not apply to their internal structure: neutron stars do not have the weak internal fields assumed in Chandra's work. This question could only be resolved by methods that can treat the weak orbital fields without making assumptions about the internal fields. Many workers developed such methods (Futamase 1983; Futamase \& Schutz 1985; Damour 1987; Will 1994; Blanchet 1996), and the result was basically that the orbits and interactions of the stars are independent of the compactness of the stars, apart from obvious effects like tidal distortions of one star by the other, and in this system such effects are ignorable.

The second kind of question was more serious for Chandra's work, which was whether it did in fact represent a valid approximation to general relativity, even for systems that were uniformly weak-field. This became an issue because of the divergent terms in Chandra's equations that we described above.

Many relativists, including Thorne (1990) and Chandra himself, did not find these infinities worrying, because they never directly entered his equations of motion. The potentials entered only in their derivatives, and Chandra knew that the

\footnotetext{
${ }^{8}$ Will writes, in a private communication, "In fact my introduction to Chandra's post-Newtonian work was in a term paper I did for [Thorne]'s general relativity course, in which I derived the EIH equations of motion by taking a suitable limit of Chandra's post-Newtonian fluid equations of motion."
} 
derivatives of the integrals were in fact finite. Later work by Kerlick (1980), working on a suggestion by Jürgen Ehlers, showed, by carefully tracking these terms, that the derivatives originally arose inside the integrals, and if they were left inside, then the contributions of these integrals to the equations of motion at $2 \mathrm{pN}$ order would be finite, as Chandra himself realised. With simple modifications, therefore, Kerlick showed that Chandra's equations can be made manifestly finite to beyond $2 \frac{1}{2} \mathrm{pN}$ order (Ehlers 1976).

Chandra's method does not, however, continue to indefinitely high order, even with derivatives left inside the integrals. Fundamentally, once radiation is present in the equations, it is impossible to place the equations of motion in an action-ata-distance, Newtonian-like form. The forces acting on fluid elements at one time are transmitted no faster than the speed of light across the fluid. The appearance of radiation reaction in the equations is the signal that retardation effects can no longer be neglected. It is impossible to construct potentials depending on the state of the fluid at a single time that fairly represent the gravitational forces at that time. Kerlick showed that the method eventually breaks down and can't be repaired. The approaches to the post-Newtonian approximation that have succeeded in going further are all formulated in terms of retarded integrals rather than Poisson-like Green functions (Will 1994; Blanchet 1996) .

This limitation on the order to which the method can be pushed has sometimes also been interpreted as casting doubt on the validity or applicability of Chandra's work. Indeed, it certainly shows that Chandra could not have developed in this way a convergent series approximation to general relativity starting at the Newtonian equations; but it seems unlikely to me that Chandra himself ever sought such a series. Chandra wanted calculational tools, the first few terms in a series that could be used to approximate weak-field systems with some accuracy. In this, he was using the (finite number of) terms that he calculated in the post-Newtonian series as an asymptotic approximation to general relativity in the limit of weak fields and slow motion.

This, it seems to me, is a justifiable claim. Approximations based on a finite number of terms can be asymptotic even when further terms in the series get unboundedly large, or indeed when one does not even have a prescription for going to further terms. There is no full proof yet that any post-Newtonian approximation is asymptotic (but see Futamese 1983 for an attempt at a proof, using restrictive assumptions), but it seems plausible in the cases of interest, and it is certainly the working assumption of all the active workers in the field. I believe that one can also make that claim with the same level of confidence for Chandra's post-Newtonian work, with the infinities regularised as described above, and for systems that have uniformly weak gravitational fields (not the Hulse-Taylor system).

Chandra himself remarked to me on more than one occasion that he felt unhappy about the controversy over the divergent terms, because it had prevented him from being given adequate credit in the world of relativity for the significance of his post-Newtonian work. I believe he may have drawn a parallel between his efforts to convince the world of astrophysics about the mass limit on white dwarfs, working 
against the prejudice of Eddington, and his foray into radiation reaction, where he encountered criticism from many established relativists. In both cases, he was a kind of outsider producing an important result and finding the reception cooler than he expected.

I do not believe that this parallel is very accurate, because Chandra was already a major scientific figure by the time he worked on the post-Newtonian approximations, his work commanding immediate attention and respect; and because the criticisms he encountered from relativists focused on real mathematical shortcomings in his work: they were not simple prejudice against an upstart outsider.

Nevertheless, I agree with him that his work has so far been under-valued. Chandra was the first person to show how, at least conceptually, the radiationreaction problem could be solved for continuous systems: he put together all the necessary ingredients, the retarded potentials, the Landau-Lifshitz pseudotensor, the near-zone expansion. He had to skate on thin ice over mathematical problems, but these turned out to be largely technical. It is easy today to forget that, at the time, there was considerable confusion in the field. One could find support in published calculations for almost any kind of radiation-reaction formula that one wanted, from the Burke-Thorne-Chandrasekhar-Esposito formula to zero reaction and even to anti-damping. ${ }^{9}$ It was, of course, precisely because of this widespread confusion, and the uncertainty about what were the reasons for such wide disagreement, that many relativists wanted to be mathematically careful, and were unhappy with the divergent integrals in Chandra's method.

Chandra did not understand this, at least partly because he had worked through the equations in such detail that he felt he had a physical feeling for the correctness of the methods he used. His was the physicist's intuition that the result was clearly (to him) correct, and if the mathematics was not fully rigourous, that was something that others could clear up. Chandra was more interested in getting to the applications, and his prompt discovery of the radiation-driven instability is itself ample proof that his time was better spent doing new physics than clearing up problems of mathematical rigour!

Chandra was a newcomer to relativity, but by applying his systematic, conservative, exhaustive methods, he became the first to provide a full description of the near-zone, post-Newtonian fluid equations through radiation-reaction order. The newcomer had accomplished, by brute force and close attention to detail, what many relativists over several decades had tried but failed to do.

\section{Wider influence: the resurgence of relativity in the $1960 \mathrm{~s}$}

There is a wider context in which the significance of Chandra's post-Newtonian work should be considered. Chandra played a key role in the movement to bring general relativity into the mainstream of physics and astrophysics. From the perspective of

\footnotetext{
${ }^{9}$ Indeed, this was true even in some papers published ten years later!
} 
1996, where general relativity plays a central role in astrophysics, where the goal of the next generation of high-energy physics theories is to unify general relativity with the other forces of physics, and where a course in general relativity is widely regarded as an essential part of a theoretical physicist's training, it may be hard to appreciate how peripheral general relativity was to physics in the 1950s. Its mathematics was unfamiliar to most physicists, even theoretical ones, and the range of physical phenomena to which it seemed relevant was small and unexciting to the leading physicists, who were trying to explain what went on in nuclear reactors and particle accelerators. Most importantly, few graduate students in physics could get any training in general relativity. If you wanted to study the subject, you went to one of a handful of departments that specialised in it. If you studied physics almost anywhere else, you ignored general relativity. In the 1950s, most ambitious physics graduate students would have shared Chandra's perception of relativity as a "graveyard" for their careers.

When changes came, many of them driven by new discoveries in astronomy, relativity was made popular, not by a group of radical young turks - as has happened in some other branches of physics - but by established physicists. The blossoming of general relativity seems to have been to a great extent initially a "top-down" phenomenon, in which several well-established physicists with broad backgrounds - among them Hermann Bondi, Bryce DeWitt, Pascal Jordan, Erwin Schrödinger, John Wheeler, and Yakov Zel'dovich - founded schools of research in relativity. They encouraged young scientists to study the subject and helped to ensure that they got their first jobs in the field. This new generation - scientists such as Bruno Bertotti, Jürgen Ehlers, James Hartle, Stephen Hawking, Igor Novikov, Roger Penrose, Dennis Sciama, Kip Thorne, James York, and many others - flourished and showed how important and relevant the field was to astronomy and to the rest of physics. I doubt if these younger people could have begun to study the subject seriously without the protection and encouragement of the established scientists.

Chandra was one of the senior figures, although he began to work in relativity later than the others I have mentioned. His move into relativity, at a time when he was the managing editor of the Astrophysical Journal, not only lent legitimacy to the subject but also provided a prestigious journal in which the younger generation, at least in the United States, could publish articles in the respectable new field of relativistic astrophysics. (All the important early papers in the Thorne-Will PPN approach appeared there, for example.) As happened so often in his career, Chandra saw that the time was right to take up a new subject; he played an important role in establishing the subject; and he obtained some of the most fundamental results. Unlike other subjects he worked on, he did not leave relativity after helping to make it important, not even after writing an important monograph (Chandrasekhar 1983). Relativity was the consuming scientific passion for the last 30 years of his life, and he was excited by new problems and new insights in it right up to the time of his death. 


\section{Acknowledgements}

I am greatly indebted to the following for helpful conversations and for supplying me with written material that I drew heavily on: Jürgen Ehlers, John Friedman, Norman Lebovitz, Bernd Schmidt, Kip Thorne, and Clifford Will. My conclusions and interpretations are, of course, entirely my own.

\section{References}

Anderson, J. L., DeCanio, T. C. 1975, Gen. Rel. Gravit., 6, 197.

Blanchet, L. 1996, "Gravitational radiation from relativistic sources", in Astrophysical Sources of Gravitational Radiation, eds., J.-A. Marck, J.-P. Lasota, (Cambridge: Cambridge University Press).

Burke, W. L. 1969, "The coupling of gravitational radiation to nonrelativistic sources", Ph.D. Thesis (California Institute of Technology, 1969).

Chandrasekhar, S. unpublished autobiographical memoirs, kindly supplied by N Lebovitz.

Chandrasekhar, S. 1964, "The Dynamical Instability of Gaseous Masses Approaching the Schwarzschild Limit in General Relativity", Astrophys. J., 140, 417-433.

Chandrasekhar, S. 1965, "The Post-Newtonian Equations of Hydrodynamics in General Relativity", Astrophys. J, 142, 1488-1512.

Chandrasekhar, S., Nutku, Y. 1969, "The Second Post-Newtonian Equations of Hydrodynamics in General Relativity", Astrophys. J, 158, 55-79.

Chandrasekhar, S. 1969, Ellipsoidal Figures of Equilibrium, (New Haven: Yale University Press).

Chandrasekhar, S. 1969, "Conservation Laws in General Relativity and in the PostNewtonian Approximations", Astrophys. J, 158, 45-54.

Chandrasekhar, S., Esposito, F. P. 1970, "The 2 $\frac{1}{2}$-Post-Newtonian Equations of Hydrodynamics and Radiation Reaction in General Relativity", Astrophys. J, 160, 153-179.

Chandrasekhar, S. 1983, The Mathematical Theory of Black Holes, (Oxford: Oxford University Press).

Damour, T. 1983, "Gravitational radiation and the motion of compact bodies", in N. Deruelle and T. Piran, Gravitational Radiation (Amsterdam, North Holland), 59-144.

Damour, T. 1987, "The problem of motion in Newtonian and Einsteinian gravity", in S W Hawking and W Israel, 300 Years of Gravitation (Cambridge: Cambridge University Press), 128-198.

Dirac, P. A. M. 1938, Proc. Roy. Soc. (London), A167, 148.

Ehlers, J., Rosenblum, A., Goldberg, J. N., Havas, P. 1976, Astrophys. J. Lett., 208, L77.

Ehlers, J. 1980, "Isolated Systems in General Relativity", Ann. N.Y. Acad. Sci., 336, 279 293.

Faulkner, J. 1971, Astrophys J, 170, L99.

Fock, V. A. 1959, Spacetime and Gravitation, (New York, Pergamon).

Futamase, T. 1983, Phys. Rev. D, 28, 2372-2381.

Futamase, T., Schutz, B. F. 1983, “The Newtonian and Post-Newtonian Approximations are Asymptotic to General Relativity", Phys. Rev. D., 28, 2363-2237.

Futamase, T., Schutz, B.F. 1985, "Gravitational Radiation and the Validity of the Far-Zone Quadrupole Formula in the Newtonian Limit of General Relativity", Phys. Rev. D, 32, p. 2557-2565. 
Hulse, R. A., Taylor, J.H. 1975, "Discovery of a pulsar in a binary system", Astrophys. J., 195, L51-L53.

Jackson, J. D. 1962, Classical Electrodynamics (New York: Wiley).

Kennefick, D. 1996, "Controversies in the history of the radiation reaction problem in general relativity", preprint.

Kerlick, G. D. 1980, Gen. Rel. Gravit., 12, 467 and 521.

Landsberg, P. T. 1982, ed., The Enigma of Time (Bristol: Hilger).

Misner, C. W., Thorne, K. S., Wheeler, J. L. 1973, Gravitation, (San Francisco: Freeman \& Co.).

Nordtvdet, K. Jr. 1968, "Equivalence Principle for Massive Bodies II. Theory", Phys. Rev., 169, 1017-1025.

Peres, A. 1960, "Gravitational radiation”, Nuovo Cim., 15, 351.

Peters, P. C. 1964, Phys. Rev., 136, 1224.

Schutz, B. F. 1980, "Statistical Formulation of Gravitational Radiation Reaction", Phys. Rev. D, 22, 249-259.

Thorne, K. S. 1969, Astrophys. J., 158, 1.

Thorne, K. S. 1990, foreword to S. Chandrasekhar, Collected Papers Vol. 5: Relativistic Astrophysics (University of Chicago Press), p. x-xx.

Trautman, A. 1958, Bull. Acad. Polon. Sci., 6, 627.

Walker, M., Will, C.M. 1980, Astrophys J, 242, L129.

Walker, M. 1984, "The quadrupole approximation to gravitational radiation", in B. Bertotti, General Relativity and Gravitation (Dordrecht: Reidel), 109-123.

Will, C. M. 1981, Theory and Experiment in Gravitational Physics, (Cambridge: Cambridge University Press).

Will, C. M. 1994, "Gravitational Waves from inspiralling compact binaries: A postNewtonian Approach”, in Relativistic Cosmology, ed., M. Sasaki (Tokyo: Universal Academy Press Inc.), pp. 83-98. 